\title{
Application of Coordinate Measuring Arm for Accurate Measurement of Child Growth
}

\author{
Marta Rępalska ${ }^{1}$, Adam Woźniak ${ }^{1}$, Marek Kulus ${ }^{2}$ \\ ${ }^{1}$ Institute of Metrology and Biomedical Engineering, Warsaw University of Technology, 8 Boboli St., 02-525 Warsaw, \\ Poland \\ ${ }^{2}$ Department of Pediatric Respiratory Diseases and Allergology, Medical University of Warsaw, 63A Żwirki $i$ Wigury St., 02- \\ 091, Warsaw, Poland
}

The article describes an approach to measure child growth using a coordinate measuring arm. For this purpose, a test set up has been built to measure the lower leg length directly on the knee surface and with the help of a plate. The use of the plate resulted in the distribution of pressure on the surface to be measured. Based on the results, the $L L V$ (a lower leg growth velocity) was determined and studies were carried out to estimate the uncertainty of this factor. The obtained $L L V$ results at $0.4 \mathrm{~mm} / \mathrm{week}$, correspond to the results of studies conducted on specialist test set-ups for knemometric measures described in the literature. The obtained results also allow recognizing periods of good health and bad health of the child.

Keywords: Dimensional metrology, coordinate measurements, child growth.

\section{INTRODUCTION}

Assessment of the total height of the body (or body length of infants) is one of the basic methods for determining the degree of maturity of the body, and in relation to age norms the proper development of the child. The standard measurements are performed by tools (stadiometer, anthropometer) with relatively low accuracy (up to $5 \mathrm{~mm}$ ), so in the prepubertal period in children (excluding the neonatal period) at a rate of growth of a few $\mathrm{cm} /$ year, measurements at shorter intervals than 6-12 months are unreliable. However, during diagnostic and treatment processes, yearly intervals, required to determine the changes in growth, are considered to be too long. Many clinical situations require the growth measurement of children every several weeks. An example is monitoring the growth of children during pharmacological treatment with potential side effects, slowing down the growth velocity of the patient.

The term "growth" is understood as physiological changes associated with an increase in the skeleton, occurring in children from birth until the end of puberty. Studies, which have been conducted so far, utilized the measuring instrument called knemometer (Greek $\eta \kappa v \eta \mu \eta-$ lower leg) [1], [2]. They were the measurements of lower leg length $L L L$ [1], which is the distance between the surface of the knee and the surface of the foot of the sitting child [2] and generally, related to the measurement of two main components - the bone and soft tissue. The choice of this part of the limb was determined by the fact that it contains a long bone (tibia), representing $48 \%$ of the length of the entire limb, always characterized by positive growth. Furthermore, a relatively small part of this section is soft tissue (e.g., in comparison to the femur), in which dynamic changes - both positive and negative - can be observed in the adipose, muscle, and skin tissue, not really reflecting the child's development [3]. Nevertheless, the measurement of the growth of the limb, in fact, a part of it, is sufficient to obtain information, required to assess the regularity of the growth process. A typical set-up for knemometric measurement is shown in Fig.1.

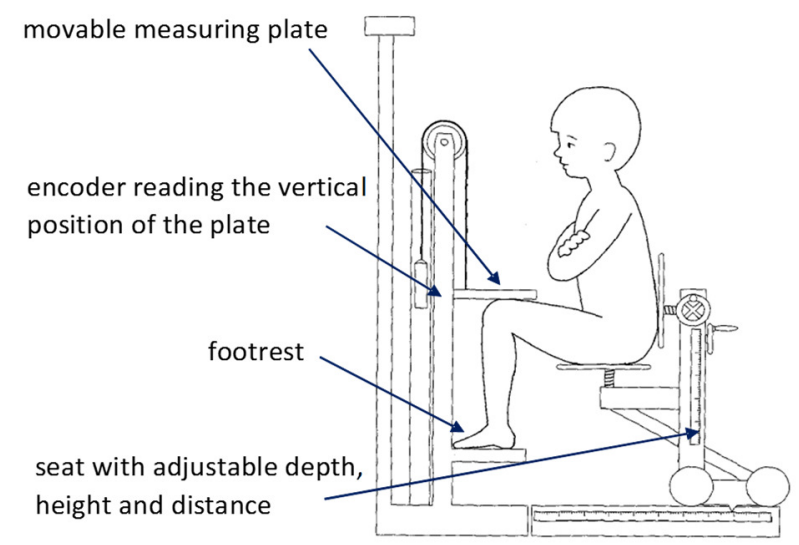

Fig.1. Typical set-up for knemometric measurement [4]. 
Before proceeding to the first examination, the child sits down freely in a special chair with his/her arms crossed on the chest. Next, the height of the chair and the depth of the seat are adjusted so as to obtain an angle of approx. $90^{\circ}$ between the thigh and lower leg so that the lower leg is perpendicular to the foot support. The measuring plate is lowered to the child's knee surface. The forward-back seat movement is then performed to find and record the highest reading - the largest distance between the foot surface and the highest point on the knee surface. Previous test set-ups for knemometric studies which have been performed until now were characterized by error (technical error) of $\sim 0.2 \mathrm{~mm}[1]$, [3]. Often, this value is also assumed for prototypes of new test set-ups, without carrying out the relevant metrological analysis [1], [4]. In general, such devices are rarely under hospital metrological supervision, current verification or periodic calibration. It should be known that the accuracy of the results depends on a number of factors. Deformations of soft tissue under the pressure, as well as the experience of the operator, are extremely important. Moreover, the realization of the measurement of a child's lower-leg growth is timeconsuming and bothersome for the little patient due to the need to stay still during the examination.

In medicine, other methods are also used to measure the geometry of the patient's body. For example, X-ray imaging methods that allow obtaining high accuracy images, [5], [6]. However, this method must be rejected in this case due to the need for frequent measurements of children. Also, noninvasive photogrammetric methods can be used in other areas, mainly related to the faulty posture evaluation, the effect of treatment and progress in patient's rehabilitation [7][9].

Nowadays, coordinate measuring techniques are going through rapid development. The first and still the most frequently used device is the coordinate measuring machine (CMM). However, over the past decade, portable variants of these devices, i.e. coordinate measuring arm, have grown in maturity, popularity, and accuracy [10]-[12]. Therefore, an attempt was made to use the coordinate measuring arm to measure child lower leg length growth and to evaluate this device from the metrological point of view.

\section{EXPERIMENTAL SET-UP FOR THE MEASUREMENT OF THE CHILDREN'S LOWER LEG LENGTH}

Experiments of measuring the lower leg length were conducted, using a specially designed test set-up, as shown in Fig.2., and the coordinate measuring arm. The test set-up has been designed to provide repeatable conditions of measurements, which are carried out during the following weeks. The increase of the length of the lower leg was determined by the measuring arm. The position of the child at the test set-up was determined by the position of the chair (1), which has adjustable seat height and depth. This solution was used to ensure a comfortable position for the child and the proper positioning of the thigh to the lower leg. The child's right foot was placed on the plate (2), the side walls of which determined the position of the ankle. In addition, the position of the right foot was blocked by the strap (3). The right angle between the surface of the thigh and the lower leg was adjusted by a plate (4) supporting the tibia. The plate (4) had a mechanism for adjustment of the position of the height and depth, which limited the possible changes of the angle between the thigh and the lower leg. The movable plate (5), which was located at the level of the knee, provided additional support of the limb and reduced a deflection of the knee joint. Adjustment ranges of the movable parts - seats and plates (4), (5), (6), were chosen according to the geometric dimensions of the child. a)

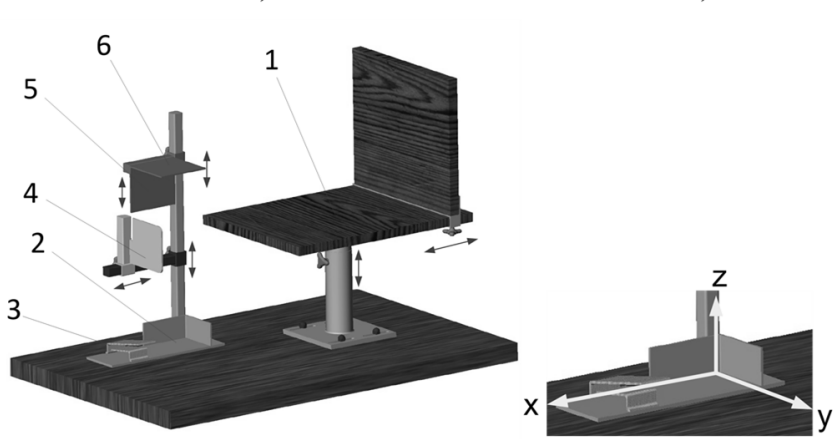

Fig.2. The test set-up for the growth measurement of the children's lower leg length: a) overall view, b) assumed coordinate system. b)

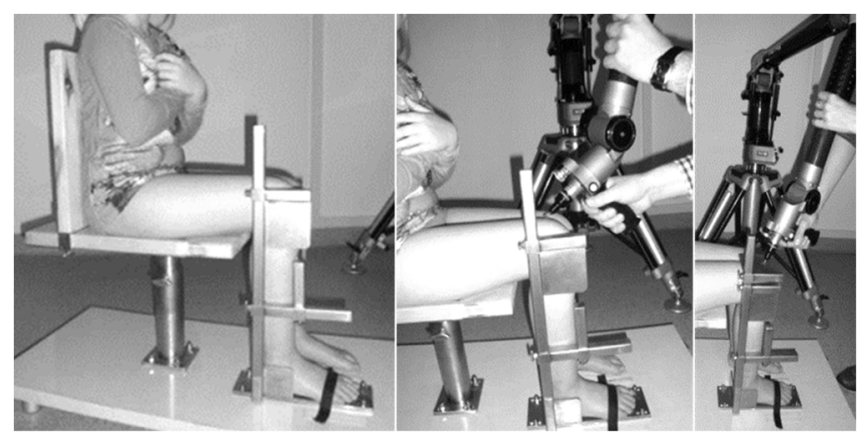

Fig.3. View of the measurement of lower-leg growth of the child using the coordinate measuring arm: a) preparation for measurement set-up, b) measurement was performed directly on the surface of the knee - configuration I, c) configuration II.

In addition to determining the position of the child, the elements of the structure were connected with the coordinate system, as shown in Fig.2.b), which was related to the coordinates of the points, designated by the measuring arm. The 7-axis measuring arm MetrisNicon MCA II, applied for measurements, was equipped with a $6 \mathrm{~mm}$ probe tip. The point repeatability test of the measuring arm, according to the ASME B89.4.22, Single Point Articulation Test [13], is $\pm 28 \mu \mathrm{m}$. The experiment concerned one child - a girl (age 5), who grows properly. The measurement of the lower leg length was carried out with the weekly time interval, always at the same time of day (in the afternoon). A single operator performed the study. The test set-up, presented in this article, enabled to measure the increase in the length of the lower leg in two strategies. The first one was carried out by setting the 
test set-up as shown in Fig.3.b) (configuration I), at which the measurement was performed directly on the surface of the knee. The first preparation for the measurements was the setting of the position of the structural elements of the test setup in the way which provided the desired position of the limb but also a comfortable position for the child, as shown in Fig.3.a)

An adjustment of the modules of the set-up to the measured object has been carried out only once. Settings of the elements have not been changed throughout the study cycle. The first step, before each measurement, was the determination of the position of the coordinate system, as shown in Fig.2.b), in which the measurements were performed. In the next stage, the child was placed on the seat, with her arms crossed on her chest, Fig.3., similarly to knemometic measurements presented in the literature [1]. During one measurement day, three measurement sessions were carried out $(j=3)$, and each session consisted of three series of measurements $(k=3)$. One series of measurements consisted of measurement of coordinates of five points on the surface of the knee $(n=5)$. These points located in the area of the topmost surface of the knee were chosen approximately, according to the subjective assessment of the operator. Then these points were marked on the skin of the child and were chosen again during the next weeks. The child would leave the test set-up between sessions; take a few steps and rest in any position.

The distance of each measuring point on the surface of the knee was determined separately in the normal direction to the $\mathrm{XY}$ plane (surface of plate 2). In the same direction (normal to the $\mathrm{XY}$ plane) the probe tip radius correction was performed. $L L L$ was evaluated as an average distance calculated from 5 measuring points.

The second applied strategy was carried out as configuration II of the test set-up, as shown in Fig.3.c), using the plate (6), Fig.2.a), lowered on the surface of the knee. During this experiment, the plate (6), Fig.2.a), was a movable and fixed element of the structure, while the other elements remained unchanged in the initial position. In configuration II, the preparation for the measurements was carried out in the same way as in configuration I. After determining the correct position of the child at the test set-up, the position of the plate (6) was set. The selected position was blocked and then it was measured using a measuring arm, Fig.3.c). After an interval during the measurements, the activities related to the adjustment of the plate to the surface of the knee were repeated and another series of measurements were performed. The measurements were repeated three times $(j=3)$ each week.

\section{RESULTS}

Fig.4. presents the measured lengths, obtained in the following weeks at the test set-up in configuration I. Although the measurements were performed at the marked points, keeping the constant position of structural elements, it was very difficult to provide the same position of the child, who would leave the test set-up for a short break. Nevertheless, taking random errors into consideration, the changes of the lower leg length, described by the determined average values $\overline{z_{i}}$ are visible.
Error bars, presented in Fig.4., correspond to the uncertainty values $u_{z i}$ characterizing the designated values $\overline{z_{i}}$. The more detailed description of the method of estimation of these values can be found below. Values, shown in Fig.4., increase in the following weeks of measurements (for $i=1 \ldots 7$ ), and the corresponding points are arranged on a straight line, with approximately a linear shape. Therefore, the obtained results reflect the increase in the length of the lower leg. Lack of marked values in weeks 4 and 5 is the result of the omission of study in that period. The "negative growth" of lower leg length, observed in weeks 8 and 9 is correlated with the reported disease and convalescence of the child at that time. This period was marked with gray color in Fig.4. Measured fragment of a limb consisted of the tibia, talus, calcaneus, ankle, knee joint, and the soft tissue of the heel and the knee [1]. Therefore, the changes observed in weeks 8 and 9 only affect the soft tissue area, despite its little share in the entire measured part of the limb. After a period of a disease, there was a break in the study. The studies were conducted again after a few weeks. Studies in weeks 16 and 17 were carried out in the child's good health. The values, obtained at that time, indicated that the process of the increase of lower leg length was just like in weeks 1 to 7 when the child was healthy as well.

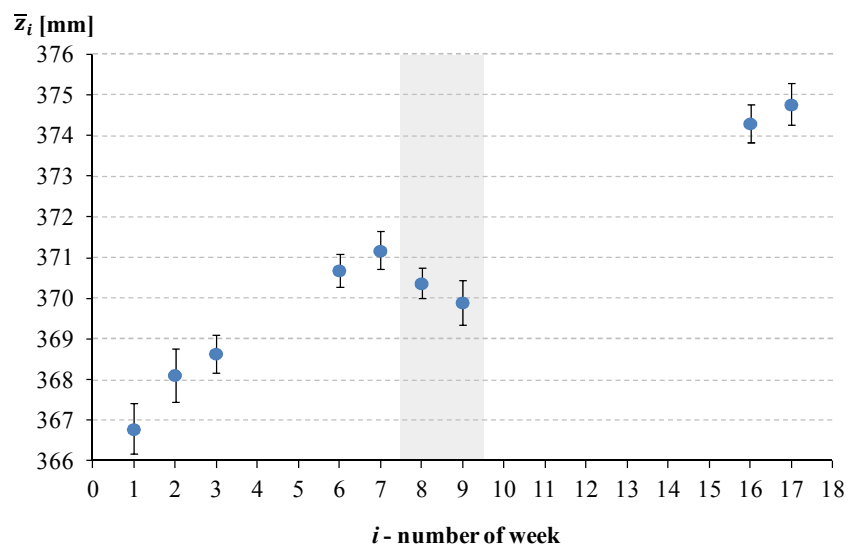

Fig.4. The results of lower leg length obtained using the set-up in configuration I.

The measurement results obtained using the test set-up in configuration II were determined similarly as in the case of configuration I, the average values $\overline{z_{i}}$, as a function of time, Fig.5. Although there were fewer results collected in this configuration than the number of results obtained in configuration I, their graphical illustration shows the periods of good health and bad health of the child.

The results obtained in weeks 1 to 7 correspond to the changes observed during the measurements performed at the test set-up in configuration I, taking into account both the nature of these changes and their quantitative range. The results of measurements conducted in weeks 8 and 9 of measurements in this configuration also confirmed the occurrence of "negative growth" of the lower leg length, which is the result of dehydration of the body (caused by vomiting and diarrhea). By contrast, the study continued during week 17 can be related to the child's good health during this period as it was at the beginning of the measuring cycle. 
Measures carried out at the test set-up in configurations I and II have produced similar results, although the process of their collection differed. Determination of the length of the lower leg, at the test set-up in configuration I required the child to maintain a constant position during the collection of measurements, namely a lot of concentration and cooperation with the operator. The applied structural solution of the test set-up in configuration II enabled to shorten the actual time of measurement. However, due to the limited capacity of the test set-up concerning the positioning of the plate (6), Fig.2., the time of preparation of the test set-up for the measurement was very long and also uncomfortable for the child.

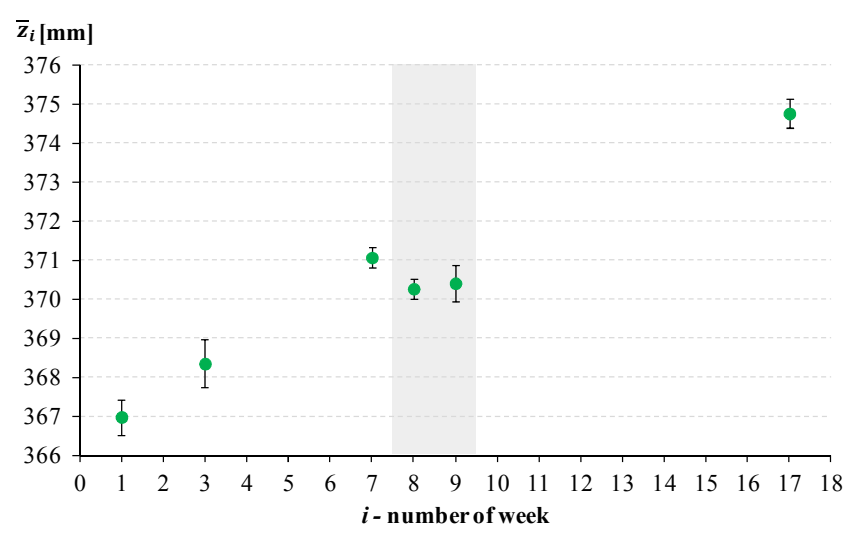

Fig.5. The results of lower leg length obtained using the set-up in configuration II.

Another problem was the deflection of the soft tissue at the point when the contact tip touches the skin. This problem occurred during the measurements performed in configuration I. Despite the light structure of the measuring arm and its free movement, there was a deflection of the soft tissue under the pressure exerted by the operator. An attempt was made to measure the quantitative assessment of the pressure exerted by the operator, performing a series of measurements of the length in the $z$-axis at one point on the surface of the knee. The result was the standard deviation of $0.4 \mathrm{~mm}$. It should be stressed that this value corresponds to the weekly increase of the lower leg length [1], [14], which shows that this issue is very important. The solution used in configuration II of the test set-up eliminated this problem during the performance of the measurements with the measuring arm. The plate (6), applied in this configuration, Fig.2., has an impact on the soft tissue. However, the pressure of the plate spreads on the larger surface of the knee.

\section{Metrological anAlysis}

Taking into consideration the coordinate values $\overline{Z_{i}}$ determined during the entire measurement cycle, a weekly increase of the lower leg length and the lower leg velocity $(L L V)$ have been determined. For this purpose, the results, obtained in the weeks 8 and 9 of measurement, associated with an abnormal state of health of the child, were excluded. The value $\overline{z_{i}}$ was characterized by the uncertainty $u_{z i}$. As it was stated above, at the test set-up with configuration I, the main sources of uncertainty included deflection of the soft tissue, repeatability of the child's position and the error of the measuring arm.

In order to determine the standard uncertainty $u_{l i}$, whose source is the deflection of the soft tissue and the error of the measuring arm, an estimate of combined standard deviations $s_{\mathrm{j}}$ was used (where $j=1 \ldots 3$ ). It was calculated from three series of measurements (one session), which is described by the following equation (1):

$$
\mathrm{u}_{1 \mathrm{i}}=\sqrt{\frac{\sum_{j=1}^{3} r_{\mathrm{j}} \cdot \mathrm{s}_{\mathrm{i}}^{2}}{\mathrm{~m}_{\mathrm{i}} \Sigma_{\mathrm{j}=1}^{3} v^{\prime}}}
$$

where: $m_{i}$ - the total number of measurements made in the $i$ th week, $v_{j}$ - degrees of freedom of the $j$-th measurement session.

Uncertainty $u_{2 i}$ associated with the positioning repeatability of the child was estimated on the basis of the range $R_{z j}$ of the value $\overline{z_{j}}$, determined in the $j$-measuring sessions. Combining uncertainties $u_{1 i}$ and $u_{2 i}$ enables the estimation of the uncertainty $u_{z i}$ of the determined values $\overline{z_{i}}$.

Measurements in configuration II of the test set-up are not dependent on the operator influence and the soft tissue deflection occurs due to the pressure of the plate (6) on the surface of the knee. The range $R_{z j}$ of the average values $\overline{Z_{j}}$, calculated from $j$-series of measurement, is characterized with repeatability of the position of the child and position errors of the plate, so it is related to uncertainty $u_{2 i}$. Deviation of the plate geometry, with its measurement at a fixed position, is expressed by the standard deviation $s_{j}$. Uncertainty $u_{1 i}$, associated with this deviation was estimated on the basis of the equation (1). Similarly, uncertainties $u_{1 i}$ and $u_{2 i}$ correspond to the assessment of the uncertainty $u_{z i}$ for the values $\overline{z_{i}}$, at the test set-up in configuration II. The calculated values $\overline{z_{i}}$ and the corresponding standard uncertainties $u_{z i}$ for configurations I and II are summarized in Table 1.

A linear regression [4], [15] was used to approximate the length of the lower leg because changes of this length were observed at the end of several weeks. Due to the fact that the uncertainties concern only points of one variable $-z$, and the values of uncertainty $u_{z i}$ which characterize it have different values in some points, weighted regression was applied. The weights of variable $\overline{Z_{i}}$ were determined on the basis of estimated uncertainties $u_{z i}$, from equation (2):

$$
w_{i}=\left(u_{z i}\right)^{-2} .
$$

The designated regression line was conducted with an omission of points corresponding to the measurement results from weeks 8 and 9 . The regression coefficient $a$, calculated from equation (3) corresponds to the lower leg velocity (LLV):

$$
a=\frac{\sum_{i=1}^{N} w_{i} \cdot \sum_{i=1}^{N} w_{i} \cdot \bar{z}_{i} \cdot i-\sum_{i=1}^{N} w_{i} \cdot \bar{z}_{i} \sum_{i=1}^{N} w_{i} \cdot i}{\sum_{i=1}^{N} w_{i} \cdot \sum_{i=1}^{N} w_{i} \cdot i^{2}-\left(\sum_{i=1}^{N} w_{i} \cdot i\right)^{2}}
$$

where $N$ is a number of analyzed values $\overline{Z_{i}}$. 
Table 1. Summary of the average values of leg length and their uncertainties, measured at the set-up in the configuration I and II.

\begin{tabular}{|c|c|c|c|c|}
\hline \multirow{2}{*}{ Week } & \multicolumn{2}{|c|}{ Configuration I } & \multicolumn{2}{c|}{ Configuration II } \\
\cline { 2 - 5 } & \multirow{2}{*}{$\bar{z}_{i}[\mathrm{~mm}]$} & $u_{z i}[\mathrm{~mm}]$ & $\bar{z}_{i}[\mathrm{~mm}]$ & $u_{z i}[\mathrm{~mm}]$ \\
\hline$i$ & & & & \\
\hline 1 & 366.79 & 0.62 & 366.98 & 0.44 \\
\hline 2 & 368.12 & 0.66 & - & - \\
\hline 3 & 368.65 & 0.47 & 368.35 & 0.61 \\
\hline 6 & 370.69 & 0.42 & - & - \\
\hline 7 & 371.18 & 0.47 & 371.07 & 0.25 \\
\hline 8 & 370.38 & 0.36 & 370.27 & 0.26 \\
\hline 9 & 369.91 & 0.54 & 370.41 & 0.46 \\
\hline 16 & 374.31 & 0.46 & - & - \\
\hline 17 & 374.77 & 0.52 & 374.76 & 0.37 \\
\hline
\end{tabular}

Determined $L L V$ for measurements at the test set-up in configuration I is $0.44 \mathrm{~mm} /$ week, while for measurements in configuration II this value is $0.46 \mathrm{~mm} /$ week. The uncertainty $u_{a}$ of the regression coefficient can be expressed as:

$$
u_{a}=\sqrt{\frac{\sum_{i=1}^{N} w_{i}}{\sum_{i=1}^{N} w_{i} \sum_{i=1}^{N} w_{i} i^{2}-\left(\sum_{i=1}^{N} w_{i} i\right)^{2}}} .
$$

Uncertainty $u_{a}$ determined for the results obtained from two configurations of the test set-up is the same and is $0.03 \mathrm{~mm} /$ week. Taking into consideration the distribution of values of the lower leg length, obtained in the $i$-th week of measurements, the standard error of estimate for the assumed linear regression model and corresponding uncertainty $u_{R}$ was determined. For this purpose, for each individual result $z_{j k n}$ the value $\left(z_{j k n}-\widetilde{z_{l}}\right)$ was determined, where $\widetilde{z_{l}}$ is the theoretical value resulting from the assumed regression model. The normality of the distribution of the determined error was confirmed. Taking into consideration the estimated value of $u_{a}$ and $u_{R}$, a combined standard uncertainty and expanded uncertainty $u_{L L V}$ was evaluated (Table 2.). Estimated expanded uncertainty $u_{L L V}$ corresponds to a coverage probability $\gamma=95.45 \%(k=2)$. The above assumption results from the normal distribution of uncertainty $u_{R}$ being the dominant component in the estimated uncertainty $u_{L L V}$.

Table 2. The lower leg velocities and their expanded uncertainties.

\begin{tabular}{|l|c|c|c|}
\hline \multirow{2}{*}{ Test set-up } & \multirow{2}{*}{$\boldsymbol{R}^{\mathbf{2}}$} & $\boldsymbol{L L} \boldsymbol{V}$ & $\boldsymbol{u}$ LLV \\
\cline { 3 - 4 } & & \multicolumn{2}{|c|}{$\mathrm{mm} / \mathrm{week}$} \\
\hline Configuration I & 0.98 & 0.44 & 0.28 \\
\hline Configuration II & 0.98 & 0.46 & 0.32 \\
\hline
\end{tabular}

\section{CONCLUSIONS}

Studies described in this article show that the observation of the increase of the lower leg length of the child can be carried out with the use of the coordinate measuring arm. The studies enabled to track the process of growth of the child during a period of several weeks and to prepare a quantitative description of the study. Furthermore, the constructed test setup enabled to perform measurements in two configurations and to compare the changes in the lower leg length, observed in these two configuration strategies. For measurements in configurations I and II of the test set-up, designated values of the lower leg velocity are $(0.44 \pm 0.28) \mathrm{mm} /$ week and $(0.46 \pm 0.32) \mathrm{mm} /$ week, respectively, which confirms that the obtained results are consistent. It should also be noted that these values are consistent with the values described in the literature $-0.4 \mathrm{~mm} /$ week [1], [3], with a standard deviation of $0.3 \mathrm{~mm} /$ week [1] - for children of the same age group. Therefore, the carried-out studies show that the results obtained from the use of industrial measuring instrument as the coordinate measuring arm, correspond to the results of studies performed at specialist test set-ups for knemometric measuring. Attempts to estimate the uncertainty of the parameter $L L V$ indicate that the contact measurements of the lower leg length are characterized by large uncertainties, often comparable to the value of the determined parameter $L L V$. This fact is mainly due to the difficulty of obtaining a repeatable position of the child at the test set-up and the deflection of the soft tissue. Nevertheless, these studies have proved that you can show the results corresponding to the periods of good health and bad health of the child from the determined values of the lower leg length. Furthermore, these studies have demonstrated the specificity of measurements connected with the fact that the tested object is a child. It should be noted that his/her age, temperament, the possibility of concentration, his/her general mental and physical state are very important factors, and although they are not metrological factors, they sometimes determine the success of the test.

Bearing in mind that the usefulness, and above all, the noninvasiveness of this kind of studies is a valuable source of information that can support the healing process, there is a need to look for other, better methods of measurements, for example, non-contact measurements. It must be taken into consideration that the accuracy of the measurements of length, in a situation where a child is the subject of the study or even a fragment of a limb, has its absolute quality limit of the obtained results.

\section{REFERENCES}

[1] Wolthers, O.D. (2010). Methodology and implications of knemometry in growth assessment of inhaled glucocorticoids. Pediatric Allergy and Immunology, 21, 190-198.

[2] Valk, I.M., Langhout Chabloz, A.M.E., Smals, A.G.H., Kloppenborg, P.W.C., Cassorla, F.G., Schutte, E.A.S.T. (1983) Accurate measurement of the lower leg length and the ulnar length and its application in short term growth measurements. Growth, 47, 53-66.

[3] Hulanicka, B, Gronkiewicz, L, Kozieł, S. (1999). Wrastanie dzieci: badanie knemometryczne. Wrocław, Poland: Zakład Antropologii PAN. (in Polish)

[4] Heuck, C., Ternowitz, T., Herlin, T., Wolthers, O.D. (1998). Knemometry in children with atopic dermatitis treated with topical glucocorticoids. Pediatric Dermatology, 15 (1), 7-11.

[5] Ryniewicz, A. (2010). Accuracy assessment of shape mapping using computer tomography. Metrology and Measurement Systems, 17 (3), 481-492. 
[6] Ryniewicz, A., Ryniewicz, A.M., Madej, T., Sładek, J., Gąska, A. (2013). Biometrological method of pelvis measurement and anatomical positioning of endoprosthesis of hip joint. Metrology and Measurement Systems, 20 (1), 17-26.

[7] Sitnik, R., Witkowski, M. (2008). Locating and tracing of anatomical landmarks based on full-field fourdimensional measurement of human body surface. Journal of Biomedical Optics, 13 (4), 044039.

[8] Witkowski, M., Glinkowski, W., Sitnik, R., Kocon, H., Bolewicki, P., Górecki, A. (2010). A four-directional body shape measurement system and its application for pectus excavatum severity assessment. In ThreeDimensional Image Processing (3DIP) and Applications, SPIE 7526.

[9] Lenar, J., Witkowski, M., Carbone, V. et al. (2013). Lower body kinematics evaluation based on a multidirectional four-dimensional structured light measurement. Journal of Biomedical Optics, 18 (5), 056014.

[10] Ostrowska, K., Gaska, A., Kupiec, R., Sładek, J., Gromczak, K. (2016). Verification of articulated arm coordinate measuring machines accuracy using LaserTracer system as standard of length. MAPANJournal of Metrology Society of India, 31 (4), 241-256.
[11] Gromczak, K., Ostrowska, K., Owczarek, D., Sładek, J. (2015). Validation of the metrological model of coordinate measuring arm using multifeature check. Advances in Science and Technology Research Journal (ASTRJ), 9 (28), 120-124.

[12] Ostrowska, K., Gaska, A., Sladek, J. (2014). Determining the uncertainty of measurement with the use of a Virtual Coordinate Measuring Arm. The International Journal of Advanced Manufacturing Technology, 71 (1-4), 529-537.

[13] The American Society of Mechanical Engineers (ASME). (2004). Methods for Performance Evaluation of Articulated Arm Coordinate Measuring Machines (CMM). Standard B89.4.22 - 2004.

[14] Wolthers, O.D. (1996). Long-, intermediate- and shortterm growth studies in asthmatic children treated with inhaled glucocorticosteroid. European Respiratory Journal, 9, 821-827.

[15] Woles, J.K.H, Milner, R.D.G. (1987). Knemometry in assessment of linear growth. Archives of Disease in Childhood, 62, 166-171.

Received January 16, 2018 Accepted September 18, 2018 